\title{
Analyzing the path of responding in maze-solving and other tasks
}

\author{
DAVID A. WASHBURN \\ Sonny Carter Life Sciences Laboratory, Georgia State University, Atlanta, Georgia
}

\begin{abstract}
Response time and accuracy are sensitive measures of overall performance but may mask underlying response strategies. For example, analysis of latency and accuracy measures produced in a computerized-maze task does not reveal whether rhesus monkeys really "solve a maze" or simply move as much as is possible toward the target, negotiating barriers through trial and error as they encounter them. Regression procedures are described for analyzing response path against several hypothetical response curves, and analyses of response path for rhesus monkeys' performance on the computerized MAZE task are presented as an illustration. The data suggest that rhesus monkeys do invoke a response strategy of solving the maze, because the observed response topography is significantly associated with the optimal path of responding. Many experimental paradigms should similarly benefit from analysis of the response paths that subjects exhibit.
\end{abstract}

Response time (RT) and accuracy are measures that are sensitive to overall performance levels but may mask the response strategies that underlie these levels. For example, the pattern swum by a rat in a water maze, the path of a chimp foraging in a field, and the trail of a cursor as it is moved to computer-generated stimuli on a screen have typically been ignored in favor of more traditional and accessible approximations via RT and accuracy measures. Response paths, however, may contain sensitive indices of the strategies that subjects use for responding.

This problem has been brought into focus in a recent series of experiments on the topic of target prediction by monkeys and humans (Washburn \& Rumbaugh, in press). Examination of the accuracy and latency of responding in a psychomotor task such as pursuit tracking does not reveal whether subjects can respond to where a target is going (i.e., predictively) or only to where a target is. As a consequence, it had been posited that only humans can be characterized as "predictor-operators" (Jaeger, 1980)-a conclusion with rather critical implications for any animal model of human tracking performance.

However, analysis of response topography in pursuit tracking and similar tasks reveals that both humans and monkeys tend to respond predictively, albeit humans do so with greater accuracy (Washburn \& Rumbaugh, in

This research was supported in part by Grant NAG2-438 from the National Aeronautics and Space Administration to Georgia State University. Additional support was provided by Grant HD-06016 from the National Institutes of Health to the Yerkes Regional Primate Research Center and by the College of Arts and Sciences of Georgia State University. The authors wish to acknowledge the theoretical and technical contributions of Duane M. Rumbaugh, Zhimin Ding, and Emil W. Menzel, Jr. Preparation of this paper was facilitated by use of the Allan M. Schrier Commemorative Library of the Language Research Center, Georgia State University, Atlanta, Georgia. Correspondence concerning this article should be sent to David A. Washburn, Department of Psychology, Georgia State University, Atlanta, GA 30303. press). That is, the observed path of responding in these tasks significantly approximates a hypothetical "path of perfect prediction." Both species, to varying degrees, tended to anticipate the movements of a target and to approach an optimal point of intersection rather than to respond toward the position actually occupied by the moving target at each point in time.

Similar problems abound in psychology. The uncertainty reflected by physical vacillation between response alternatives, the distances and directions that constitute wayfinding, and the strategies-or lack thereof-that underlie various search behaviors are clearly important. However, the need to analyze response topography is undermined by numerous difficulties, typically resulting in the attempt to capture these effects through a combination of response latency, time, duration, accuracy, and anecdote. The resultant loss of data may have obscured many important findings.

Chief among the difficulties that accompany the analysis of response topography is how to quantify the behavior. This problem reflects not only the need to find a coordinate system for coding the response path, but in many cases uncertainty regarding the proper unit of analysis (i.e., how to break continuous responding down into discrete units). However, some types of research produce response topography that is relatively free of these difficulties. The computerized tasks used at our laboratory in research with rhesus monkeys (Macaca mulatta), orangutans (Pongo), chimpanzees (Pan troglodytes and paniscus), and humans (both normally developing and mentally retarded children, as well as adults) are an example.

Movement on a computer screen is apparent rather than real; that is, stimuli do not move but rather flash from place to place, with each "jump" providing only the illusion of movement. Each jump is thus associated with a discrete location on the screen and can be treated as a trial, in that it represents an opportunity to choose or 
change directions. A series of jumps--the path of responding-can be represented by an array of $x, y$ coordinates. Thus, computerized tasks easily provide the level of "path data" that are more difficult to obtain in less automated testing (maze solving by rats, traditional choice RT in humans, etc.).

\section{ANALYSIS OF RESPONSE PATH}

The general strategy being advanced is one of comparing the observed path of responding-the actual stream of $x, y$ coordinates in time that capture each trial-with one or more hypothetical paths, such as the predictive path or the tracking path in the Washburn and Rumbaugh (in press) study. These hypothetical paths should be derived from theory, and take the form "given a cursor location of $x_{C}, y_{C}$ and a target location of $x_{P}, y_{P}$, where would the subject move if using strategy $S_{1}$ ? Where would the subject move if using strategy $S_{2}$ ? Where in fact did the subject move?"

Several means exist for determining the amount of variance in observed paths of responding that can be associated with various predictors, but multiple correlation and regression techniques are particularly well suited for this type of analysis. To illustrate this type of analysis, response path data from an experiment on computer-task MAZE solving will now be examined.

\section{METHOD}

\section{Subjects}

Ten rhesus monkeys (Macaca mulatta), ranging in age from 2 to 9 years, were tested. Only 1 of the monkeys was a female. Two of the animals, Abel and Baker, have extensive test histories that have been documented elsewhere (e.g., Rumbaugh, Richardson, Washburn, Savage-Rumbaugh, \& Hopkins, 1989; Washburn, Hopkins, \& Rumbaugh, 1991). The remaining animals were trained with the procedures and tasks that had been developed for Abel and Baker. All monkeys were unrestrained within their home cages and were not deprived of food or water for purposes of testing.

\section{Apparatus}

Hardware. The monkeys were trained and tested with the Language Research Center's Computerized Test System, which consists of a battery of software tasks and the computer hardware required to administer them (see Washburn \& Rumbaugh, 1992). An XT-compatible microcomputer recorded all data and presented all stimuli on a color monitor. Each subject responded to these computergenerated stimuli by manipulating a joystick, which in turn controlled the movements of a cursor (a 1-cm white " +") on the screen. Audio feedback was presented through an external speaker/amplifier, and fruit-flavored chow pellets (P. J. Noyes, Lancaster, NH) were delivered following each successfully completed trial.

Software. The task used in the present experiment was the MAZE task, in which subjects had to negotiate computer-graphic barriers on the screen in order to bring the cursor into contact with a small, stationary target (a blue square approximately $1 \mathrm{~cm}$ in size) ${ }^{1}$ The mazes used in this experiment, depicted in Figure 1, were drawn with PC-Storyboard and saved as bit-mapped (.PIC) files; they filled the computer screen completely. Many of the mazes used in this experiment were inspired by the barrier problems designed by Hebb and Williams (1946). However, the MAZE task was designed to be used with any maze or barrier problem that could be drawn in black and white and BLOADed from a file.

The task itself was a rather straightforward modification of the CHASE task (Rumbaugh et al., 1989), in which the subject must bring the cursor into contact with a moving target. A predrawn maze template was first loaded by the task. Any white area on the screen (other than the cursor) was interpreted by the task as a wall of the maze. Movements of the joystick produced isomorphic movements of the cursor unless such movements would position the cursor off the screen or place the cursor into contact with a maze wall. The MAZE task can be administered in two modes: Random or Set-up. In Random mode, the target and cursor are positioned randomly at the beginning of each trial (with the restraint that neither could be positioned on a maze wall or atop the other). In Set-up mode, one or more pairs of starting positions can be specified for the target and the cursor, with the computer choosing randomly between these potential position pairs at the start of each trial. Although the task will permit a moving target, only stationary targets were used in this experiment.

Collision between cursor and target was registered as a correct response, and it resulted in the delivery of audio feedback and a pellet. For each trial, RT was recorded for the interval between the first joystick movement and collision with the target. Any trial in which the cursor was moved but then remained stationary for $120 \mathrm{sec}$ was aborted ("dropped out"), and the trial was readministered (thus, if an animal "got stuck and gave up," a new trial would

Training maze templates

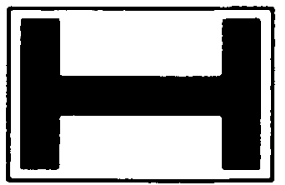

1

Test-maze templates

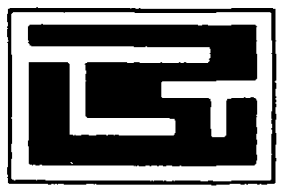

1

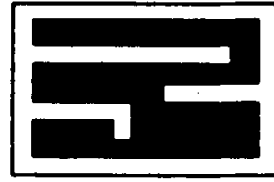

3

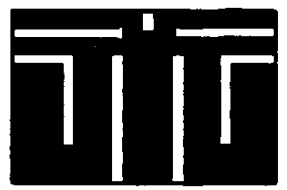

2

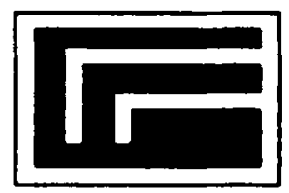

2

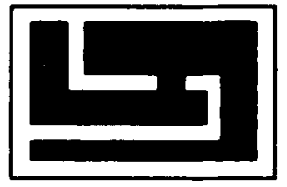

4

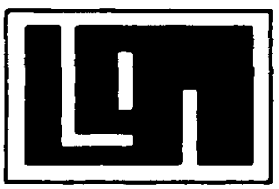

5

Figure 1. Maze templates used in training and in this experiment. 


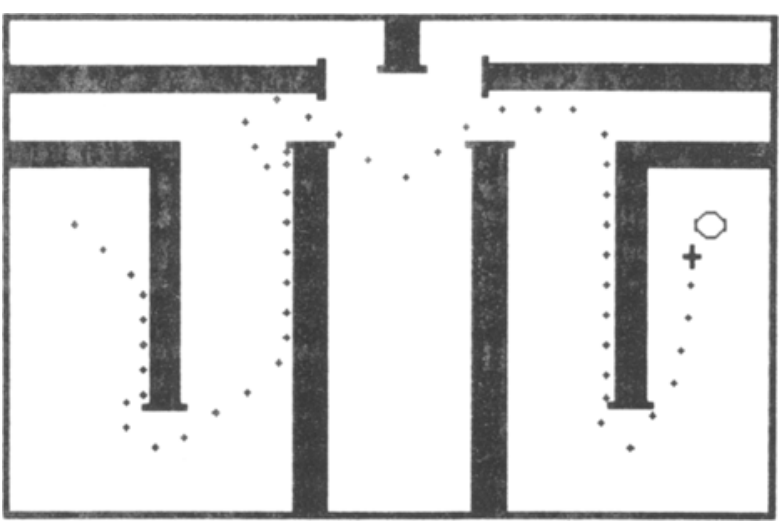

Figure 2. Depiction of responding in a MAZE trial. The dots represent the path of the cursor, showing frequent contact with the MAZE walls.

automatically be presented). A 5-sec intertrial interval separated the trials.

\section{Procedure}

Training. Prior to the beginning of this experiment, each subject was trained to respond in the MAZE task with the use of the two training templates depicted in Figure 1. In this training, the cursor and target were located randomly within one of the two training mazes. The subjects were trained on each maze until the target was contacted in less than $5 \mathrm{sec}$, averaged across 200 trials.

During this training period, each subject learned that maze walls were barriers that could not be traversed, but rather had to be circumvented to reach the target. However, even skilled subjects were observed usually to collide with intervening maze walls en route to the target (see an example in Figure 2). Several potential reasons for this behavior were posited. Subjects might have collided with maze walls in an attempt to minimize travel distance around them. Alternatively, subjects might have exercised the general strategy of moving directly toward the target, and finding a way around the barriers only when absolutely necessary. According to the latter suggestion, the subjects might not have "solved the maze" at all, but rather circumvented maze walls through trial and error only when thwarted from moving directly toward the target. From the RT and accuracy data, it was impossible to discern between these potential explanations. This tendency of the subjects to contact maze walls, and our inability to explain such a tendency without analyzing response paths, prompted the present study.

Testing. The five test-maze templates in Figure 1, selected because they cannot be solved by moving directly toward the target, were used in this experiment. Each animal was given a block of 100 trials on each of the mazes, although the order of maze administration was randomized between animals. The cursor always began each trial in the lower right corner of the maze, with the target located in the upper left corner, irrespective of which maze template was being tested (i.e., the MAZE task was used in Set-up mode).

In addition to standard RT data, the computer maintained the coordinate-by-coordinate record of the cursor's movements for each trial. Trials that were dropped out were not counted against the required number for completion, although the response path taken on these trials was recorded and used in analysis. Thus, each "path data" file contained all of the information required to reconstruct every response made by each subject for each trial.

Analysis. At least two hypothetical response strategies (or algorithms for generating hypothetical paths) could be devised for the MAZE task, against which the observed path of responding was analyzed. First, the subject might attempt to move directly toward the target at each opportunity, moving randomly when absolutely prevented from advancing toward the target (the "target path"). Alternatively, the subject could move along the path of optimal maze solution, without direct regard for the location of the target (the "optimal path"'). Of course, other theory-derived paths and combinations of algorithms could be hypothesized, but for the purposes of illustrating the analysis of interest, these two hypothetical paths will suffice.

For analysis, each $x, y$ value, representing each movement of the cursor, was first translated into a single value. Any single unit of measure, such as a distance (using the Pythagorean theorem) or angle, could be used with comparable results. Because cursor movements are a reflection of joystick angle of deflection, the angle of movement was computed for each $x, y$ coordinate in the present analysis [using the formula: angle $=$ (arctangent of the slope) $180 / \pi$, where the slope = rise/run; see Appendix]. Thus, for each movement during a trial, the angle at which the cursor moved on the screen was computed (relative to the horizon, so that moving directly left was coded as $0^{\circ}$, moving straight up was computed to $90^{\circ}$, and so forth). This angle of movement was compared with (1) the angle that would have been taken if the subject had moved directly toward the target, and (2) the angle that would have been taken if
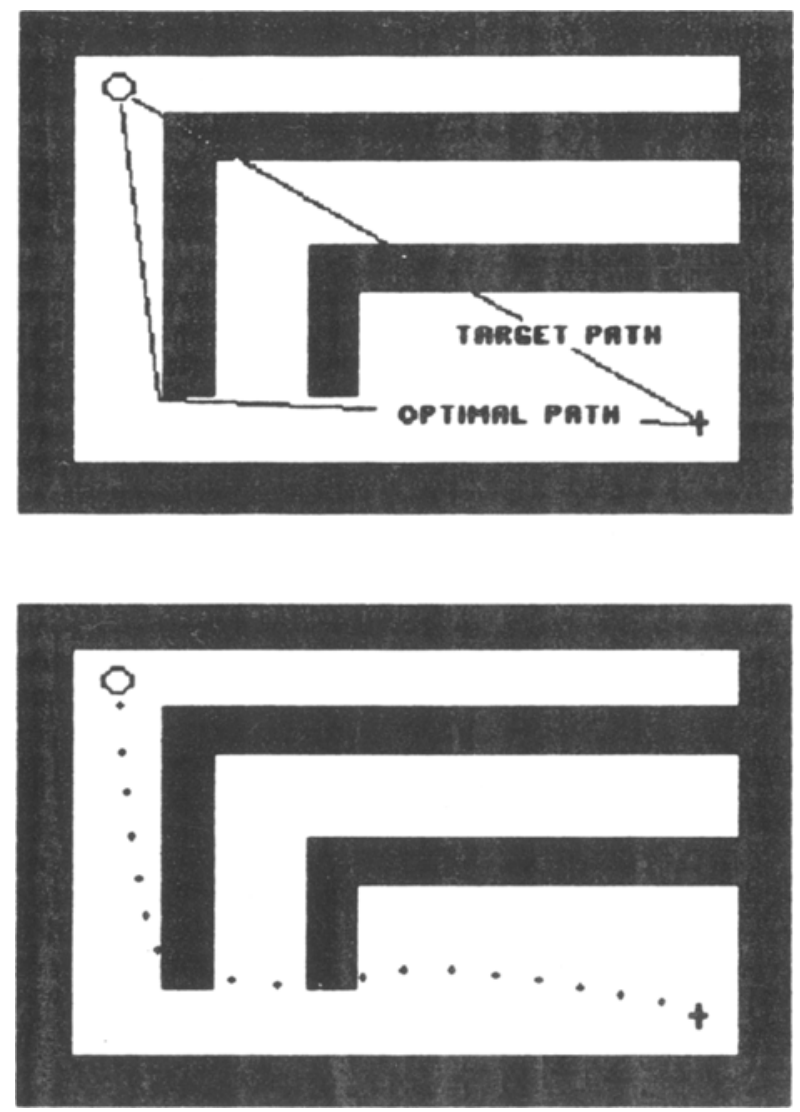

Figure 3. The initial target and optimal path values for TestMaze 2 (top). Using these and subsequent values, a "best fit" curve is plotted (bottom) from the equation $y=($ Target path) $(-.33)+$ (Optimal path)(.88) +22 . Note that the cursor could not actually cross the walls in the MAZE task. 
the subject had moved toward the optimal solution of the maze. For each move on the screen, then, three different angles were computed, reflecting a movement-by-movement comparison of potential response strategies.

For each maze, portions of the line of optimal solution were identical to the path generated by moving directly toward the target. To avoid spurious correlations, the degree of association between the target path and the optimal path was partialed out of each correlation between observed and hypothetical paths. The resultant semipartial correlation coefficients ( $r_{\text {observed, (optimal tarzet) }}$ and $r_{\text {observed, }}$ (target.optimal)) reflect the degree to which the observed path of responding was uniquely associated with the optimal and the target paths, respectively.

\section{RESULTS}

For each of the 500 trials per animal in this experiment, an average of 109.5 data points (i.e., moves, which were recoded as angles of movement) were generated. Consequently, over one-half million total data points were available for analysis from this sample. Because substantial learning occurred across the 100 trials that each animal experienced with a particular maze pattern (revealed by the response times as well as response paths), results of analyzing only the first 10 correctly completed trials with each maze will be discussed. ${ }^{2}$

The observed paths of responding were observed to be highly correlated with the two hypothetical paths in this experiment $\left(r_{\text {observed, target, optimal }}=.91, p<.01\right)$. Thus, over $80 \%$ of the variance in observed response path was associated with target-directed and optimal-solution strategies. The significant majority of this association, however, was uniquely associated with the hypothetical "optimal" path. The semipartial correlation coefficient for observed and optimal paths was $r_{\text {observed,(optimal.target) }}=$ $.58(p<.01)$; the degree to which response topography was uniquely associated with the hypothetical target path was only $r_{\text {observed,(target. optimal) }}=.05(p>.05)$.

Multiple regression analyses produced an equation for drawing a line of best fit for each of the mazes. These equations revealed the relative weights placed on the target and optimal strategies, as well as the walls of the maze normally contacted. One such curve, averaged across animals for for Test-Maze 2, is presented in Figure 3.

\section{DISCUSSION}

These data suggest that rhesus monkeys tend to respond along the optimal path of responding in simple mazes rather than utilize a basic strategy of always moving toward the target. Collisions of the cursor and the maze wall appear to result from imprecision in joystick manipulation along this optimal path rather than from attempts to exercise a direct route to the target-the present analyses do not reveal a significant unique association between cursor movements and target location. Of course, each of the present mazes afforded a fairly obvious solution, requiring little planning. Further manipulation of the complexity of maze problems (e.g., increasing the number and location of walls) and the complexity of the potential hypothetical paths (e.g., combining strategies and including distance from the walls as a factor) should permit identification of how far in advance rhesus monkeys and other species can "plan ahead" to solve maze problems.

These conclusions could not follow from analysis of MAZE RT data alone. Of course, one could approximate the present analyses by coding accuracy for the MAZE task-as indeed was done in the experiment that inspired the present study (Hebb \& Williams, 1946). Additionally, collisions with the walls, entrances to cul-de-sacs, pauses, and changes in direction could be recorded, providing composite measures of path accuracy that approach the level of detail used in the present analysis. The analysis of response path itself could in fact be seen as this type of statistic, with responses coded along a continuous range of values for a large but finite number of measures. For problems in which only one or two categories of topography are interesting, the rough coding of accuracy across several measures may well suffice. However, for any behavior in which a wide range of response paths may be observed, with each one codable and comparable to the others, the advantages of the present analysis as a complement to traditional measures should be obvious.

\section{REFERENCES}

HeBb, D. O., \& WiLliams, K. (1946). A method of rating animal intelligence. Joumal of General Psychology, 34, 59-65.

JAEGER, R. J. (1980). Predictive capabilities of nonhuman operators in a manual control system. Perceptual \& Motor Skills, 51, 759-764.

Rumbaugh, D. M., Richardoon, W. K., Washburn, D. A., SavageRumbaugh, E. S., Hopkins, W. D. (1989). Rhesus monkeys (Macaca mulatta), video tasks, and implications for stimulus-response spatial contiguity. Joumal of Comparative Psychology, 103, 32-38.

Washburn, D. A., Hopkins, W. D., Rumbaugh, D. M. (1991). Perceived control in rhesus monkeys (Macaca mulatta): Enhanced video-task performance. Journal of Experimental Psychology: Animal Behavior Processes, 17, 123-129.

Washburn, D. A., RUmbaugh, D. M. (1992). Testing primates with joystick-based automated apparatus: Lessons from the Language Research Center's Computerized Test System. Behavior Research Methods, Instruments, \& Computers, 24, 157-164.

Washburn, D. A., Rumbaugh, D. M. (in press). A comparative assessment of psychomotor performance: Target prediction by humans and macaques. Joumal of Experimental Psychology: General.

\section{NOTES}

1. The MAZE task is a part of the Language Research Center's Computerized Test System. For information on availability, contact the author.

2. Results from the entire sample of data, and indeed from analysis of only Trial 1 data, differ in degree but not in general from the presented findings. 


\section{APPENDLX}

For analysis of response angle, given Cartesian coordinates where $\left(x_{i}, y_{i}\right)$ is the location of a stimulus at time $i$ and $\left(x_{i-1}, y_{i-1}\right)$ is the previous location of that stimulus:

IF $x_{i}<>x_{i-1}$

$$
\text { slope }=\left(y_{i}-y_{i-1}\right) /\left(x_{i}-x_{i-1}\right)
$$

angle $^{\circ}=$ ATN(slope $) \cdot 180 / 3.141592653$

IF $x_{i}=x_{i-1}$ 'adjust for occasions of no slope

THEN IF $y_{i}>y_{i-1}$ THEN angle $^{\circ}=90$ ELSE angle ${ }^{\circ}=270$

IF $x_{i}>x_{i-1}$ THEN angle $^{\circ}=180-$ angle $^{\circ}$

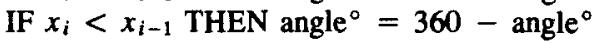

'adjust for direction

IF angle ${ }^{\circ}>259$ then angle ${ }^{\circ}=$ angle $^{\circ}-360$ 'relative to left horizon

IF angle ${ }^{\circ}<0$ then angle ${ }^{\circ}=$ angle $^{\circ}+360$ 'adjust angle 'for $360^{\circ}$

Note-If angle of movement is employed as the dependent measure, care must be exercised to ensure that it is translated accurately on a linear scale. For instance, an observed angle of $5^{\circ}$ differs equally from a target path of $25^{\circ}$ and an optimal path of $345^{\circ}$. To ensure proper analysis, the latter value must be coded as $-15^{\circ}$. 